\title{
Rank-Based Whale Optimization Algorithm for Solving Parameter Optimization of Solar Cells
}

\author{
Jeng-Shyang Pan, Jenn-Long Liu, and En-Jui Liu
}

\begin{abstract}
The efficiency of solar cells is usually determined by the characteristic curve of voltage-current relationship. The objective function for the solar cell design is often nonlinear and multimodal. Accordingly, a global search algorithm is desired to solve the objective function. This study applies a lately developed metaheuristic algorithm, named whale optimization algorithm (WOA) which has good global search capability, to solve the objective function for estimating the undetermined parameters of solar cells. Furthermore, this paper proposed a rank-based WOA with a nonlinear rank-based selection pressure model to replace the random selection process used in the original WOA at the stage of searching for prey. A tournament selection based WOA is also presented for comparison in this work. The performance of the rank-based WOA is assessed by testing six benchmark functions. From the experiments, the present rank-based WOA outperforms the original and tournament selection based WOAs in optimizing benchmark functions. Moreover, the proposed Rank-based WOA is applied to the parameter optimization of solar cell problem. The optimal solutions and parameters obtained using the rank-based WOA will be compared with those of using the original and tournament selection based WOAs.
\end{abstract}

Index Terms-Rank-based whale optimization algorithm; nonlinear rank-based selection pressure model; parameter optimization; solar cell problem.

\section{INTRODUCTION}

The greenhouse effect caused by a large amount of carbon dioxide, methane, nitrous oxide, ozone, chlorofluorocarbons and other gases is remarkable, resulting in sustained warming and extreme weather occurrence on the Earth. In July this year, countries in Europe suffered from unusual high-temperature heatwaves due to the polar heat vortex. The temperature in Nordic countries such as Norway, Sweden, the Netherlands and Germany were as high as 30 to 35 degrees Celsius, and the temperature difference reached as high as 20 degrees when compared with previous summers. In addition, tens of thousands of people fled their homes in California due to the forest fires caused by unusual high temperatures. The heatwaves have spurred wildfires that have claimed at least 80 lives in Greece, burned many houses down and melted electrical wires in California, and forced Sweden to call for

Manuscript received October 7, 2018; revised May, 1, 2019.

Jeng-Shyang Pan is with the College of Information Science and Engineering, Fujian University of Technology, Fuzhou 350118, Fujian, China (e-mail: jengshyangpan@fjut.edu.cn).

Jenn-Long Liu is with the Department of Information Management, I-Shou University, Kaohsiung 84001, Taiwan (e-mail: jlliu@isu.edu.tw).

En-Jui Liu is with the Department of Power Mechanical Engineering, National Tsing Hua University, Hsinchu 30013, Taiwan (e-mail: adw2579@gmail.com). emergent help from Swedish Air Force to drop 500-pound GBU-49 guided bombs in the burning forest to help fight the disaster. As a result, the issue of reducing the emissions of exhaust gas and saving energy has been highly valued and vigorously promoted by the governments of most countries in the world over the years. In addition to reducing energy waste, finding alternative energy is also an important policy for governments. Considering the cause of the current use of thermal power is one of the major causes of air pollution, so the power generation using clean energy such as wind power, hydroelectricity and solar energy is indispensable to reduce the emissions of exhaust gas.

In clean energy, solar power is one of the most promising renewable energy sources because of the abundant sources of solar light. According to the assessment report of SolarPower Europe [1], the installed capacity of solar power systems worldwide has increased significantly every year. Global solar installations increased by $98.9 \mathrm{GW}$ (or 29\%) in 2017. China and India account for $63 \%$ of the total demand for solar energy in the year. In the solar power system, solar panels are composed by connecting series of solar cells, or named photovoltaic cells, to provide a variety of electricity demands. Considering the efficiency of solar cells is based on the current-voltage characteristic curve (i.e. I-V curve), the solar cell design requires accurate modeling to test. There are two steps to model the solar cells: mathematical model formula and accurate estimation of undetermined parameters. At present, two equivalent electronic circuit models, single diode (SD) and double diode (DD) [2], are commonly used to simulate the behavior of solar cells. Both models need to estimate all their parameters, such as the current generated by solar, the saturation current and ideal factor of the diode, the series resistance, etc. The SD model needs to estimate five parameters, while the DD model needs to estimate seven ones. The undetermined parameters of SD or DD model can be obtained by fitting experimental data. Therefore, the maximum power point of the solar cell can be obtained from the voltage-power curve after determining the parameters of the applied model.

Generally, the estimation methods for undetermined parameters of solar cell can be divided into two categories: deterministic and heuristic methods. Deterministic methods, such as Least Square Method [3], Lambert W-functions [4], and Iterative Curve Fitting [5] are fast in estimation, but they are very sensitive to the initial solutions. Namely, these techniques are often trapped in the local optimum. In recent years, many heuristic algorithms have been proposed to solve the optimization problem of solar cell for parameter estimation because of their global searching ability. In the relevant literature, several heuristic methods have been 
applied to the optimization of solar cell parameter estimation, including Genetic Algorithm (GA) [6], Particle Swarm Optimization (PSO) [7], Artificial Bee Colony (ABC) [8], Whale Optimization Algorithm (WOA) [9] and so on. The results obtained using these heuristic algorithms are usually better than those obtained using the deterministic ones. Among these heuristic algorithms, WOA is the latest metaheuristic algorithm which is inspired by the observation of whales' hunting behavior. Although the WOA has been successfully applied to many kinds of optimization problems [10], but like other basic heuristic algorithms, it tends to converge to the local optimal solution early. Therefore, this paper proposes an improved version of WOA and then applies it to the parameter estimation of solar cells.

The remainder of this paper is organized as follows. Section II describes the double diode model of solar cell. Section III presents the principles and formulations of the original WOA, tournament selection based WOA, and rank-based WOAs. Section IV reports the computational results of benchmark functions and parameter optimization of double diode model of solar cell. Finally the main conclusions are given in Section V.

\section{Double Diode Model of Solar Cell}

Generally, single diode (SD) model is the most commonly used for simulating the electronic circuits of solar cells. The equivalent circuit of SD model is displayed in Fig.1. The formulations for simulating this model are relatively simple. As shown in Fig. 1, the model uses a diode to shunt the photogenerated current $\left(I_{p h}\right)$ and a resistor in series with the diode. In addition, the actual diode has a non-ideal factor that affects the voltage-current relationship of the element.

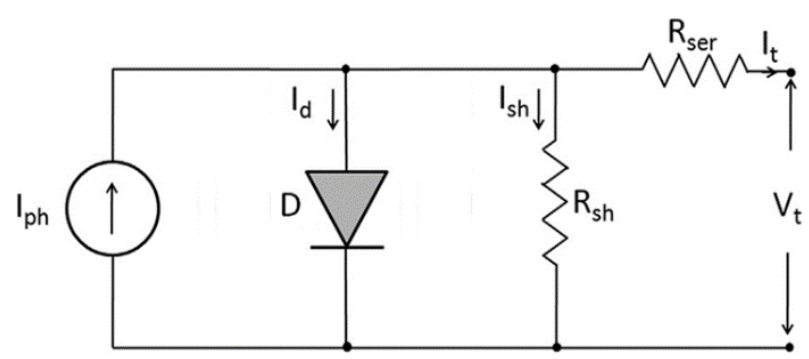

Fig. 1. Single diode model of solar cell.

where $I_{p h}$ is the current source (or photogenerated current); $I_{t}$ and $V_{t}$ are the net current and voltage, respectively; $I_{d}$ is saturation current; $I_{s h}$ is leakage current caused by shunt resistance $R_{s h}$. To further consider the accuracy of the I-V curve, this study uses a double diode (DD) model. This model is more complex than $\mathrm{SD}$, but it can present the internal phenomena of parameters related to solar cells. The configuration of the equivalent circuit for DD model is displayed in Fig. 2. The equivalent circuit of DD model for the solar cell system has designed with a current source in parallel with two diodes. Also, there are series resistance and shunt resistance in the DD model. The net current $\left(I_{t}\right)$ of the solar cell system for DD model can be expressed as follows:

$$
I_{t}=I_{p h}-I_{d 1}-I_{d 2}-I_{s h}
$$

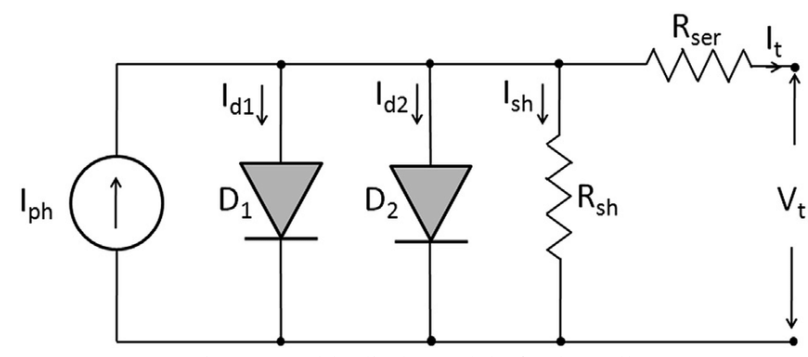

Fig. 2. Double diode model of solar cell.

where $I_{p h}$ is the current source (or photogenerated current); $I_{t}$ and $V_{t}$ are the net current and voltage, respectively; $I_{d l}$ and $I_{d 2}$ are diffusion and saturation currents, respectively; $I_{s h}$ is leakage current caused by shunt resistance $R_{s h}$. In order to properly model the solar cell, Shockley's diode equation [11] is applied in this work. The formulations of currents $I_{d l}$ and $I_{d 2}$ related to net voltage $V_{t}$ can be expressed as

$$
\begin{aligned}
& I_{d 1}=I_{s d 1}\left\lfloor\exp \left(\frac{q\left(V_{t}+R_{\text {ser }} \times I_{t}\right)}{n_{1} \times k \times T}\right)-1\right\rfloor \\
& I_{d 2}=I_{s d 2}\left\lfloor\exp \left(\frac{q\left(V_{t}+R_{s e r} \times I_{t}\right)}{n_{2} \times k \times T}\right)-1\right\rfloor
\end{aligned}
$$

Therefore, (1) can be rewritten as

$$
\begin{aligned}
& I_{t}=I_{p h}-I_{s d 1}\left\lfloor\exp \left(\frac{q\left(V_{t}+R_{s e r} \times I_{t}\right)}{n_{1} \times k \times T}\right)-1\right\rfloor- \\
& I_{s d 2}\left[\exp \left(\frac{q\left(V_{t}+R_{s e r} \times I_{t}\right)}{n_{2} \times k \times T}\right)-1\right]-\frac{V_{t}+R_{s e r} \times I_{t}}{R_{s h}}
\end{aligned}
$$

where electronic quantity of electricity is $q=1.602 \times 10^{-19} \quad$ (coulombs); Boltzmann constant is $k=1.380 \times 10^{-23}(\mathrm{~J} / \mathrm{K}) ; T$ is the cell temperature. Therefore, there are seven undetermined parameters in the DD model: (1) the current of light generation $\left(I_{p h}\right),(2)$ the reverse saturation current of diffusion $\left(I_{s d l}\right)$, (3) the reverse saturation current of recombination $\left(I_{s d 2}\right),(4)$ the ideal factor of diffused diode $\left(n_{1}\right)$, (5) the ideal factor of recombined diode $\left(n_{2}\right),(6)$ the series resistance $\left(\mathrm{R}_{\text {ser }}\right)$, and (7) shunt resistance $\left(R_{s h}\right)$. The above parameters can be estimated from the I-V curve of solar cells. To determine the parameters of DD model, the WOAs are applied. The optimization process is performed by minimizing the objective function devised by the root mean square error (RMSE) of net current between the computed values of $\left(I_{t}\right)_{\text {comp }}$ and experimental data of $\left(I_{t}\right)_{\text {exp }}$ at $N$ different conditions of $V_{t}$. Therefore, the objective function $(f)$ used in this work is defined as

$$
R M S E=\sqrt{\frac{1}{N} \sum_{n=1}^{N}\left|\left(I_{t}\right)_{n, \text { comp }}-\left(I_{t}\right)_{n, \exp }\right|^{2}}
$$




\section{ORIGINAL AND PROPOSED WOAS}

\section{A. Original Whale Optimization Algorithm}

A mentioned in Section I, Whale Optimization Algorithm (WOA) is a new swarm intelligence algorithm proposed by Marjalili and Lewis in 2016. Whales are highly intelligent mammals in the ocean. Bubble-net attacking method is often used when whales are in group hunting. As a flock of krill or small fishes is found, the whales will first dive about 10-15 meters below the surface of the ocean and then swim around the fish in a circle or 9-shaped path below the flock. The whales will eventually hunt in a spiral direction toward the sea level. Based on this peculiar hunting behavior of humpback whales, three stages of whale swarm algorithm are inspired as follows: encircling around prey, searching for prey, and bubble-net attacking by shrinking around prey and swimming with spiral path for hunting. In order to capture prey effectively, the hunting pattern of the WOA includes two important mechanisms: exploitation and exploration. The basic whale swarm algorithm is described as below.

\section{1) Stage of encircling around prey}

First, whales observe and memorize the locations of the prey, and then encircle around them. When encircling the prey, whales update their location towards the best location obtained so far. Therefore, the model in the stage of encircling prey can be formulated as follows [10]:

$$
\vec{X}(t+1)=\vec{X}^{*}(t)-\vec{A} \cdot \vec{D} ; \quad \vec{D}=\left|\vec{C} \cdot \vec{X}^{*}(t)-\vec{X}(t)\right|
$$

where $\vec{X}(t)$ represents position vector and $\vec{X}^{*}(t)$ represents the best solution found so far; $t$ is the index of current iteration; $\vec{A}$ and $\vec{D}$ are coefficient vectors; (.) denotes an element-by-element multiplication and || denotes the absolute value. The vectors $\vec{A}$ and $\vec{C}$ are generated based on random functions as follows:

$$
\vec{A}=2 \vec{a} \cdot \vec{r}-\vec{a} ; \quad \vec{C}=2 \cdot \vec{r}
$$

Since the coefficient $\vec{r}$ is a random vector in $[0,1]$, then the ranges of vectors $\vec{A}$ and $\vec{C}$ are in the intervals $[-\vec{a}, \vec{a}]$ and $[0,2]$, respectively. The value of $\vec{a}$ is specified to decrease linearly from 2 to 0 from the start to the end of iteration.

\section{2) Stage of searching for prey}

The vector $\vec{A}$ shown in (6) also can be applied to the stage of search for prey when whales explore the possible position of prey. In the basic WOA, the position of a whale is updated by selecting a whale randomly from the population [10].

$$
\vec{X}(t+1)=\vec{X}_{\text {rand }}(t)-\vec{A} \cdot \vec{D} ; \quad \vec{D}=\left[\vec{C} \cdot \vec{X}_{\text {rand }}(t)-\vec{X}(t)\right]
$$

where $\vec{X}_{\text {rand }}$ represents a position vector of whale selected by randomness. The value of $\vec{A}$, represented by $|\vec{A}|$ exists two cases: greater than 1 or less than 1 . When $|\vec{A}|>1$, the search agent will move far away from a reference whale and the search emphasizes exploration to allow the WOA performing a global search; When $|\vec{A}|<1$, the search agent will update the position according to the current best solution and the search emphasizes exploitation to allow the WOA performing a local search.

\section{3) Stage of bubble-net attacking}

In the stage of bubble-net attacking, whales swim around the prey within shrinking circle as well as simultaneously move along a spiral-shaped path to form distinctive bubbles along a 9-shaped path [10]. Two types of behavior in the stage of bubble-net attacking include shrinking around prey and spiral path swimming for hunting

Shrinking Around Prey: As mentioned above, the value of $\vec{a}$ decreases linearly from 2 to 0 . Since the range of vectors $\vec{A}$ is in $[-\vec{a}, \vec{a}]$, so the value of $\vec{A}$ decreases over the course of iterations. Therefore, the shrinking encircling mechanism can be achieved by using (6).

Spiral Path Swimming for Hunting: In this phase, each whale updates its position according to a spiral-shaped path. The spiral-shaped equation between the positions of whale and prey can be expressed as follows:

$$
\vec{X}(t+1)=\vec{D}^{\prime} \cdot e^{b l} \cdot \cos (2 \pi l)+\vec{X}^{*}(t) ; \vec{D}^{\prime}=\left|\vec{X}^{*}(t)-\vec{X}(t)\right|
$$

where $b$ is a constant for defining the logarithmic shape and $l$ is a random number in $[-1,1] ; \vec{D}^{\prime}$ represents the distance between positions of prey (i.e. the best solution found so far, denoted by $\left.\vec{X}^{*}(t)\right)$ and whale (denoted by $\vec{X}(t)$ ).

To model this simultaneous behavior at the stage of bubble-net attacking, Mirjalili and Lewis assumed that there is a probability of selection with 0.5 to choose either carrying out the shrinking encircling mechanism or the spiral-shaped movement to update the position of whale during iterations The simultaneous behavior in the stage of bubble-net attacking is modeled as follows:

$$
\vec{X}(t+1)=\left[\begin{array}{ll}
\vec{X}^{*}(t)-\vec{A} \cdot \vec{D} & \text { if } \operatorname{rand}()<0.5 \\
\vec{D}^{\prime} \cdot e^{b l} \cdot \cos (2 \pi l)+\vec{X}^{*}(t) & \text { otherwise }
\end{array}\right]
$$

\section{B. Tournament Selection Based WOA}

As shown in (8), the selection of $\vec{X}_{\text {rand }}$ in the original WOA is used based on random numbers. However, the random selection may slow down the convergence rate of WOA. Therefore, Mafarja and Mirjalili proposed the tournament selection instead of random selection for $\vec{X}_{\text {rand }}$ to improve the performance of WOA. The tournament selection is a simple and effective approach to give a selection pressure when picking solutions from the population. These solutions are compared against each other. The solution with high fitness value will be selected. The fitness value for a solution $i$, fitness $s_{i}$, can be obtained from the value of the objective function as follows. 


$$
\text { fitness }_{i}=\left\{\begin{array}{l}
1 /\left(1+f_{i}\right), \text { if } f_{i} \geq 0 \\
1+\left|f_{i}\right|, \text { if } f_{i}<0
\end{array}\right\}
$$

As shown in (8), the exploration in the original WOA depends on changing the position of each search agent according to a randomly selected solution. The selection of $\vec{X}_{\text {rand }}$ is modified by the tournament selection as follows:

$$
\vec{X}_{\text {rand }}(t)=\left\{\begin{array}{ll}
\vec{X}_{i}(t), & \text { if } \text { fitness }_{i}>\text { fitness }_{j} \\
\vec{X}_{j}(t), & \text { otherwise }
\end{array}\right\}
$$

\section{Proposed Rank-Based WOA}

In the presented rank-based WOA, The selection of $\vec{X}_{\text {rand }}$ is based on a probability ( $p^{\text {rank }}$ ) which is designed based on the rank of fitness of solutions. The rank equaling to 1 means that the fitness value is maximum for all agents, whereas the rank equaling to $N$ means the value of fitness is minimum. Here $N$ is the number of agents (i.e. whales). The nonlinear rank-based selective pressure model is formulated as

$$
p_{k}^{\text {rank }}=\frac{1}{1-(1-q)^{N}} \times q \times(1-q)^{\text {rank-1 }}
$$

with satisfying the constraint: $\sum_{k=1}^{N} p_{k}^{r a n k}=1$. The index $k$ denotes the assigned number of an agent after sorting fitness values for all agents. The value of parameter $q$ is a positive real in $[0,1]$. The larger value of $q$ implies a stronger selective pressure for the value of probability. In the present rank-based WOA, the value of $q$ is 0.5 . It means that the best agent has $50 \%$ chances to be selected as the reference agent. The accumulated probability for an agent $k$ can be calculated as follows:

$$
A_{\text {Cprob }}=\sum_{m=1}^{\text {rank }} p_{k}^{\text {rank }}, \quad \text { for } k=1,2, \ldots, N
$$

Since all agents are sorted according to their values of fitness. The agent $\vec{X}_{\text {rand }}$ can be determined by (15).

$$
\vec{X}_{\text {rand }}(t)=\left\{\begin{array}{ll}
\vec{X}_{1}(t) & \text { if rand }() \leq A \text { Cprob }_{1} \\
\vec{X}_{k}(t) & \text { if } A \text { Cprob }_{k-1}<\operatorname{rand}() \leq \text { ACprob }_{k}
\end{array}\right\}
$$

\section{RESUlTS AND DisCUSSION}

\section{A. Experiments on Benchmark Functions Using Whale Optimization Algorithms}

In this work, six standard benchmark functions for minimization were evaluated to assess the performance of the prosed WOA. The six benchmark functions were Sphere, Rosenbrock, Griewank, Rastrigin, Ackley, and Schwefel 2.26, as listed in Table I. The optimal solutions for Functions 1-6 all listed in Table I. In this work, the population size of agents was 30 . Three scales of space size with $D=30, D=50$, and $D=100$ were studied. In addition, the maximum number of generations and independent runs were 500 and 30 for all experiments, respectively. Table II compares the best mean solutions (Mean), standard deviation (Std), and best (Best) solutions obtained using the original, tournament selection based, and rank-based WOAs for the six benchmark functions. The better solution was marked with bold font in the table. As listed in Table II(a), the solutions obtained using the rank-based WOA were significant better than those obtained using the original and tournament selection based WOAs for medium-scale problems for the cases of $D=30$. Except for Function 2, the proposed rank-based WOA can all achieve optimal solutions effectively. In generally, the present rank-based WOA outperforms the original and tournament selection based WOAs in terms of Mean, Std, and Best. Similarly, the solutions obtained using the rank-based WOA were better than those obtained using the original and tournament selection based WOAs for large-scale problems for the cases of $D=50$ and $D=100$, listed in Tables II(b) and (c). Experimental results revealed that the rank-based WOA significantly outperforms the original and tournament selection based WOAs for solving the medium- and large-scale problems.

TABLE I: SIX BENCHMARK FUNCTIONS USED FOR THE EVALUATION OF WOAS

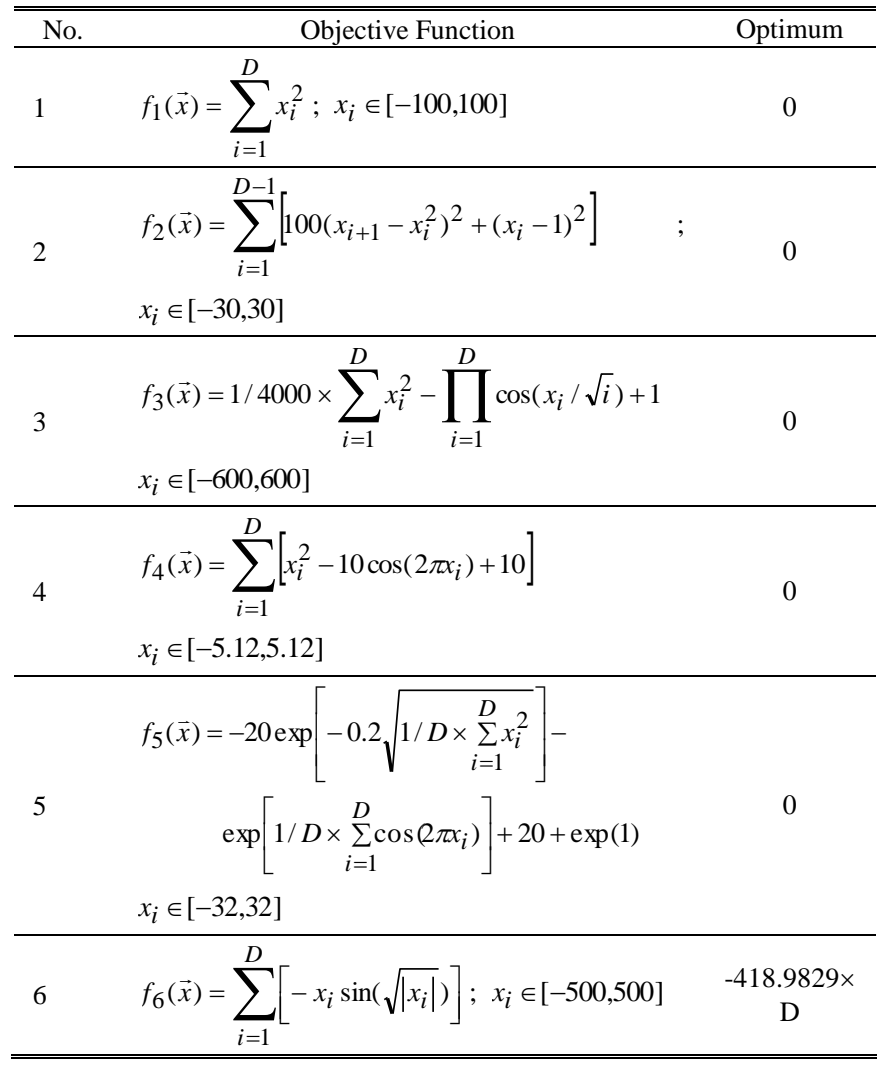

Figs. 3(a)-(f) plot the convergence histories of solutions evaluated using the three WOAs for the cases of $D=50$. Clearly, the rank-based WOA had the best convergence rate. The convergence histories of solutions were plotted using a logarithmic y-axis for Functions 1-5 to reveal the variations 
among the convergence rate and optimal solutions. Similarly, the rank-based WOA got the best performance for the all cases of $D=30$ and $D=100$ in terms of convergence rate. From the computational results, the rank-based WOA effectively found the global optima with fast convergence, and is a promising algorithm for solving complex optimization problems of engineering.

TABLE II: COMPARISONS OF SOLUTION FOR THE CASES OF (A) $D=30$, (B) $D=50$, AND $D=100$

(a) $D=30$

\begin{tabular}{|c|c|c|c|c|}
\hline Fun. & Sol. & Original WOA & $\begin{array}{c}\text { Tournament } \\
\text { WOA }\end{array}$ & $\begin{array}{c}\text { Rank-based } \\
\text { WOA }\end{array}$ \\
\hline \multirow{3}{*}{$f_{1}(\vec{x})$} & Mean & $1.2079 \mathrm{e}-075$ & $4.1873 \mathrm{e}-082$ & 8.9531e-094 \\
\hline & $(\mathrm{Std})$ & $(4.014 \mathrm{e}-075)$ & $(1.510 \mathrm{e}-081)$ & $(4.628 \mathrm{e}-093)$ \\
\hline & Best & $1.3277 \mathrm{e}-083$ & $2.6051 \mathrm{e}-092$ & $5.7836 \mathrm{e}-106$ \\
\hline \multirow{3}{*}{$f_{2}(\vec{x})$} & Mean & $2.7945 e+001$ & $2.7902 \mathrm{e}+001$ & $2.8726 \mathrm{e}+001$ \\
\hline & $(\mathrm{Std})$ & $(5.120 \mathrm{e}-001)$ & $(5.434 \mathrm{e}-001)$ & $(6.399 \mathrm{e}-002)$ \\
\hline & Best & $2.6944 e+001$ & $2.7072 \mathrm{e}+001$ & $2.8486 \mathrm{e}+001$ \\
\hline \multirow{3}{*}{$f_{3}(\vec{x})$} & Mean & $1.9491 \mathrm{e}-002$ & $4.6756 \mathrm{e}-003$ & $0.0000 \mathrm{e}+000$ \\
\hline & $(\mathrm{Std})$ & $(5.890 \mathrm{e}-002)$ & $(2.517 \mathrm{e}-002)$ & $(0.000 \mathrm{e}+000)$ \\
\hline & Best & $0.0000 \mathrm{e}+000$ & $0.0000 \mathrm{e}+000$ & $0.0000 \mathrm{e}+000$ \\
\hline \multirow{3}{*}{$f_{4}(\vec{x})$} & Mean & $3.7895 \mathrm{e}-015$ & $1.8947 \mathrm{e}-015$ & $0.0000 \mathrm{e}+000$ \\
\hline & (Std) & $(2.040 \mathrm{e}-014)$ & $(1.020 \mathrm{e}-014)$ & $(0.000 \mathrm{e}+000)$ \\
\hline & Best & $0.0000 e+000$ & $0.0000 \mathrm{e}+000$ & $0.0000 \mathrm{e}+000$ \\
\hline \multirow[t]{3}{*}{$f_{5}(\vec{x})$} & Mean & $4.0856 \mathrm{e}-015$ & $3.3750 \mathrm{e}-015$ & $2.6645 \mathrm{e}-015$ \\
\hline & $(\mathrm{Std})$ & $(2.311 \mathrm{e}-015)$ & $(2.452 \mathrm{e}-015)$ & $(1.999 \mathrm{e}-015)$ \\
\hline & Best & $8.8817 \mathrm{e}-016$ & $8.8817 \mathrm{e}-016$ & 8.8817e-016 \\
\hline \multirow[t]{3}{*}{$f_{6}(\vec{x})$} & Mean & -1.0043 .5771 & -10955.2143 & -12535.7906 \\
\hline & $(\mathrm{Std})$ & $(1.570 \mathrm{e}+003)$ & $(1.636 \mathrm{e}+003)$ & $(1.040 \mathrm{e}+002)$ \\
\hline & Best & -1.2569 .4866 & -1.2569 .4866 & -12569.4866 \\
\hline \multicolumn{5}{|l|}{ (b) $D=50$} \\
\hline Fun. & Sol. & Original WOA & $\begin{array}{l}\text { Tournament } \\
\text { WOA }\end{array}$ & $\begin{array}{l}\text { Rank-based } \\
\text { WOA }\end{array}$ \\
\hline \multirow[t]{3}{*}{$f_{1}(\vec{x})$} & Mean & $2.2870 \mathrm{e}-073$ & $5.9029 \mathrm{e}-080$ & $4.1095 \mathrm{e}-091$ \\
\hline & $(\mathrm{Std})$ & $(1.070 \mathrm{e}-072)$ & $(2.125 \mathrm{e}-079)$ & $(1.924 \mathrm{e}-090)$ \\
\hline & Best & $8.4991 \mathrm{e}-088$ & $8.8804 \mathrm{e}-091$ & 3.8291e-106 \\
\hline \multirow{3}{*}{$f_{2}(\vec{x})$} & Mean & $4.8256 \mathrm{e}+001$ & $4.8196 \mathrm{e}+001$ & $4.8592 \mathrm{e}+001$ \\
\hline & $(\mathrm{Std})$ & $(4.101 \mathrm{e}-001)$ & $(4.321 \mathrm{e}-001)$ & $(4.984 \mathrm{e}-002)$ \\
\hline & Best & $4.7004 \mathrm{e}+001$ & $4.7121 \mathrm{e}+001$ & $4.8412 \mathrm{e}+001$ \\
\hline \multirow[t]{3}{*}{$f_{3}(\vec{x})$} & Mean & $0.0000 \mathrm{e}+000$ & $8.6386 \mathrm{e}-003$ & $0.0000 \mathrm{e}+000$ \\
\hline & $(\mathrm{Std})$ & $(0.000 e+000)$ & $(3.241 \mathrm{e}-002)$ & $(0.000 \mathrm{e}+000)$ \\
\hline & Best & $0.0000 \mathrm{e}+000$ & $0.0000 \mathrm{e}+000$ & $0.0000 \mathrm{e}+000$ \\
\hline \multirow{3}{*}{$f_{4}(\vec{x})$} & Mean & $0.0000 \mathrm{e}+000$ & $1.8947 \mathrm{e}-015$ & $0.0000 \mathrm{e}+000$ \\
\hline & $(\mathrm{Std})$ & $(0.000 e+000)$ & $(1.020 \mathrm{e}-014)$ & $(0.000 \mathrm{e}+000)$ \\
\hline & Best & $0.0000 \mathrm{e}+000$ & $0.0000 \mathrm{e}+000$ & $0.0000 \mathrm{e}+000$ \\
\hline \multirow[t]{3}{*}{$f_{5}(\vec{x})$} & Mean & $4.3224 \mathrm{e}-015$ & $4.0856 \mathrm{e}-015$ & $2.7829 \mathrm{e}-015$ \\
\hline & $(\mathrm{Std})$ & $(2.335 \mathrm{e}-015)$ & $2.486 \mathrm{e}-015)$ & $(1.772 \mathrm{e}-015)$ \\
\hline & Best & $8.8817 \mathrm{e}-016$ & 8.8817e-016 & 8.8817e-016 \\
\hline \multirow[t]{3}{*}{$f_{6}(\vec{x})$} & Mean & -17814.6899 & -16730.7935 & -20668.5483 \\
\hline & $(\mathrm{Std})$ & $(2.984 \mathrm{e}+003)$ & $(2.780 \mathrm{e}+003)$ & $(5.779 e+002)$ \\
\hline & Best & -20946.0673 & -20942.8954 & -20949.1444 \\
\hline \multicolumn{5}{|l|}{ (c) $D=100$} \\
\hline Fun. & Sol. & Original WOA & $\begin{array}{c}\text { Tournament } \\
\text { WOA }\end{array}$ & $\begin{array}{c}\text { Rank-based } \\
\text { WOA }\end{array}$ \\
\hline \multirow[t]{3}{*}{$f_{1}(\vec{x})$} & Mean & $1.1015 \mathrm{e}-070$ & $1.1983 \mathrm{e}-077$ & $7.4625 \mathrm{e}-093$ \\
\hline & $(\mathrm{Std})$ & $(5.916 \mathrm{e}-070)$ & $(6.028 \mathrm{e}-077)$ & (3.061e-092) \\
\hline & Best & $2.1023 \mathrm{e}-081$ & $3.5599 \mathrm{e}-089$ & $7.4691 \mathrm{e}-107$ \\
\hline$f_{2}(\vec{x})$ & Mean & $9.8193 e+001$ & $9.8031 \mathrm{e}+001$ & $9.5241 \mathrm{e}+001$ \\
\hline & $(\mathrm{Std})$ & $(2.126 \mathrm{e}-001)$ & $(2.981 \mathrm{e}-001)$ & $(1.592 \mathrm{e}+001)$ \\
\hline & Best & $9.7590 \mathrm{e}+001$ & $9.7320 \mathrm{e}+001$ & $9.4822 \mathrm{e}+000$ \\
\hline$f_{3}(\vec{x})$ & Mean & $0.0000 \mathrm{e}+000$ & $7.5105 \mathrm{e}-003$ & $0.0000 \mathrm{e}+000$ \\
\hline & $(\mathrm{Std})$ & $(0.000 e+000)$ & $(4.044 \mathrm{e}-002)$ & $(0.000 e+000)$ \\
\hline & Best & $0.0000 \mathrm{e}+000$ & $0.0000 \mathrm{e}+000$ & $0.0000 \mathrm{e}+000$ \\
\hline$f_{4}(\vec{x})$ & Mean & $3.7895 \mathrm{e}-015$ & $3.7895 \mathrm{e}-015$ & $0.0000 \mathrm{e}+000$ \\
\hline & $(\mathrm{Std})$ & $(2.040 \mathrm{e}-014)$ & $(2.040 \mathrm{e}-014)$ & $(0.000 \mathrm{e}+000)$ \\
\hline & Best & $0.0000 \mathrm{e}+000$ & $0.0000 \mathrm{e}+000$ & $0.0000 \mathrm{e}+000$ \\
\hline$f_{5}(\vec{x})$ & Mean & $4.4408 \mathrm{e}-015$ & $4.4408 \mathrm{e}-015$ & $3.4935 e-015$ \\
\hline & $(\mathrm{Std})$ & $(2.426 \mathrm{e}-015)$ & $(2.426 \mathrm{e}-015)$ & $(2.037 \mathrm{e}-015)$ \\
\hline & Best & $8.8817 \mathrm{e}-016$ & $8.8817 \mathrm{e}-016$ & $8.8817 \mathrm{e}-016$ \\
\hline$f_{6}(\vec{x})$ & Mean & -34251.2608 & -35907.2261 & -40879.2148 \\
\hline & $(\mathrm{Std})$ & $(5.825 \mathrm{e}+003)$ & $(5.357 \mathrm{e}+003)$ & $(1.891 \mathrm{e}+003)$ \\
\hline & Best & -41892.0912 & -41891.0898 & -41898.2887 \\
\hline
\end{tabular}

(a) $f_{1}(\vec{x})$

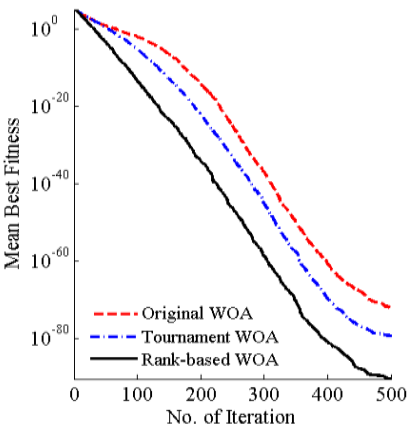

(c) $f_{3}(\vec{x})$

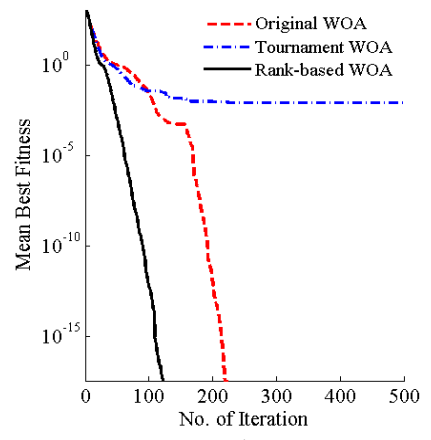

(e) $f_{5}(\vec{x})$

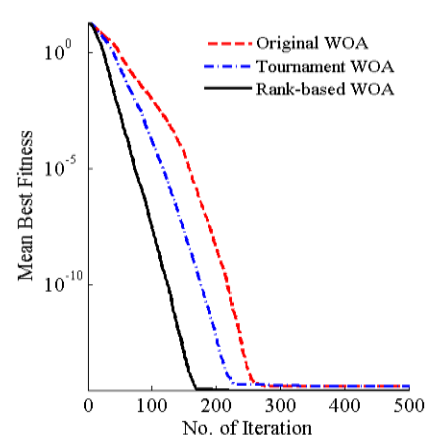

Fig. 3. Comparisons of convergence histories obtained using the original, tournament selection based and rank-based WOAs for the cases of $D=50$.

\section{B. Parameters Estimation of Double Diode Model}

As described in Section II, the net current $\left(I_{t}\right)$ in the DD model of solar cell can be obtained by solving (4). Therefore, the undetermined parameters in (4) can be acquired by using the original, tournament selection based, and rank-based WOAs. The search ranges for the seven parameters applied in the WOAs were listed as in Table III.

TABLE III: THE SEARCH RANGES FOR THE SEVEN PARAMETERS

\begin{tabular}{ll}
\hline \hline Parameter & Range \\
\hline$R_{s e r}(\Omega)$ & {$[0,0.5]$} \\
$R_{s h}(\Omega)$ & {$[0,100]$} \\
$I_{p h}(\mathrm{~A})$ & {$[0,1]$} \\
$I_{s d l}(\mu \mathrm{A})$ & {$[0,1]$} \\
$I_{s d 2}(\mu \mathrm{A})$ & {$[0,1]$} \\
$n_{l}$ & {$[1,2]$} \\
$n_{2}$ & {$[1,2]$} \\
\hline \hline
\end{tabular}

The cell temperature was set to be $33^{\circ} \mathrm{C}$. The number of agents was 50, and the number of iteration for a run was 200 . Independent runs are performed 30 times to analyze the 
robustness of the algorithm. Fig. 4 shows the I-V characteristic curves of the experimental data and computational results obtained using the three WOAs. Clearly, the computed values using the three algorithms are quite close to the experimental ones. Fig. 5 depicts the comparison of power- $\mathrm{V}$ characteristic curves of the experimental data and computational results obtained using the three WOAs. The results also showed that the calculated values using the three WOAs are also quite close to the experimental values. From the solution displayed in Fig. 5, the maximum power was $0.3100545(\mathrm{~W})$ when the net voltage was $0.459(\mathrm{~V})$. Fig. 6 demonstrated that the convergence rate and solution accuracy obtained using the rank-based WOA is better than those of the original and tournament WOAS.

Furthermore, Table IV lists the comparisons of net currents between calculated and experimental data. The comparisons revealed that the calculated values obtained using the three WOAs were all agree well with the experimental data. Table $\mathrm{V}$ is the optimal values of parameter and objective function calculated by using the three WOAs. From the comparison of RSME, the order of optimal objective function values obtained using the three WOAs was represented by rank-based WOA>original WOA>tournament selection based WOA. The comparison revealed show that the proposed WOA outperformed the other two kinds of WOAs.

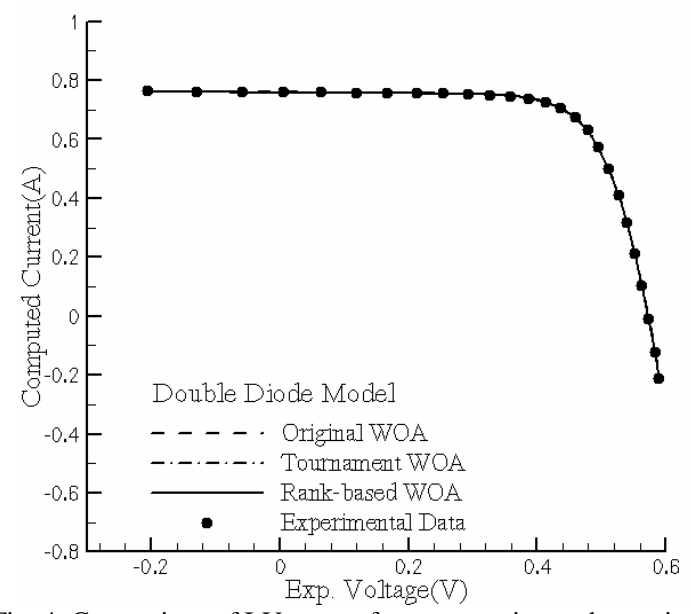

Fig. 4. Comparison of I-V curves for computation and experiment.

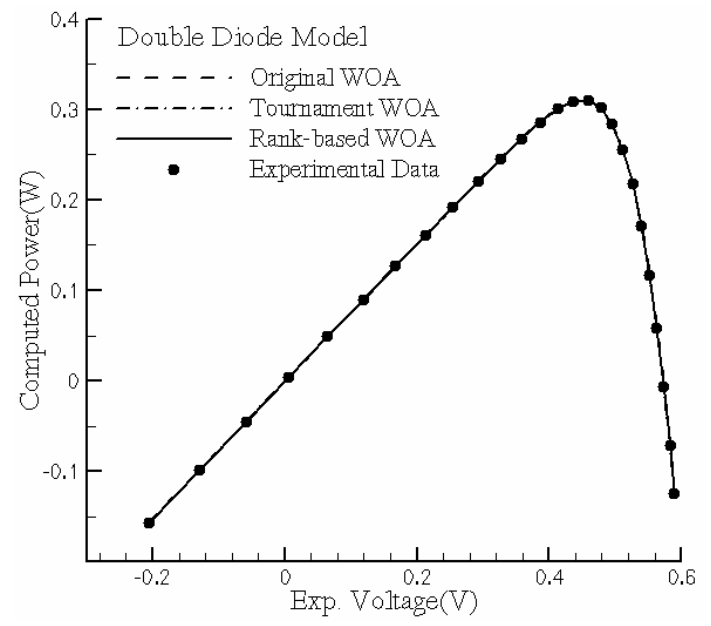

Fig. 5. Comparison of Power-V curves for computation and experiment.
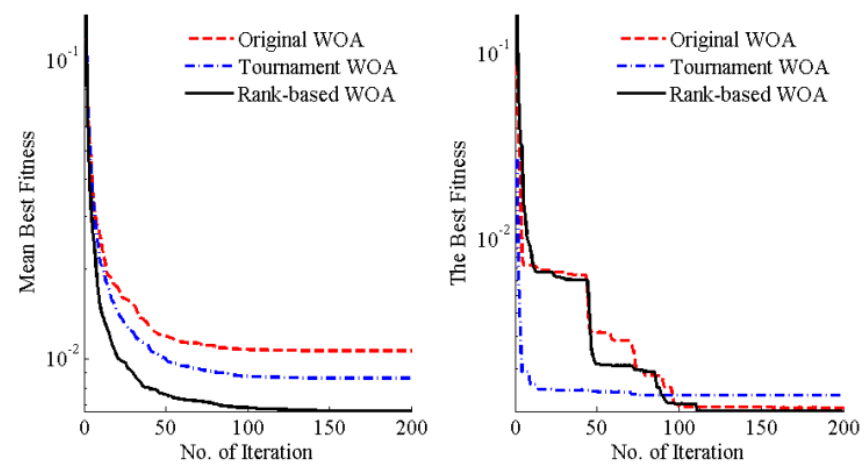

Fig. 6. Comparison of convergence histories obtained using the three WOAs

TABLE IV: COMPARISONS OF NET CURRENTS FOR COMPUTATION AND EXPERIMENT

\begin{tabular}{|c|c|c|c|c|}
\hline \multicolumn{2}{|c|}{ Experimental Data $\left(\mathrm{V}_{\mathrm{t}}-\mathrm{I}_{\mathrm{t}}\right)$} & Basic WOA & $\begin{array}{l}\text { Tournament } \\
\text { WOA }\end{array}$ & $\begin{array}{l}\text { Rank-based } \\
\text { WOA }\end{array}$ \\
\hline $\mathrm{V}_{\mathrm{t}}(\mathrm{V})$ & $\mathrm{I}_{\mathrm{t}}(\mathrm{A})$ & \multicolumn{3}{|c|}{ Computed $\mathrm{I}_{\mathrm{t}}(\mathrm{A})$} \\
\hline-0.2057 & 0.7640 & 0.7626 & 0.7607 & 0.7621 \\
\hline-0.1291 & 0.7620 & 0.7616 & 0.7599 & 0.7611 \\
\hline-0.0588 & 0.7605 & 0.7608 & 0.7592 & 0.7601 \\
\hline 0.0057 & 0.7605 & 0.7599 & 0.7585 & 0.7592 \\
\hline 0.0646 & 0.7600 & 0.7592 & 0.7579 & 0.7584 \\
\hline 0.1185 & 0.7590 & 0.7585 & 0.7573 & 0.7577 \\
\hline 0.1678 & 0.7570 & 0.7578 & 0.7568 & 0.7569 \\
\hline 0.2132 & 0.7570 & 0.7571 & 0.7562 & 0.7562 \\
\hline 0.2545 & 0.7555 & 0.7563 & 0.7554 & 0.7553 \\
\hline 0.2924 & 0.7540 & 0.755 & 0.7543 & 0.7540 \\
\hline 0.3269 & 0.7505 & 0.7528 & 0.7523 & 0.7518 \\
\hline 0.3585 & 0.7465 & 0.7488 & 0.7484 & 0.7477 \\
\hline 0.3873 & 0.7385 & 0.7415 & 0.7413 & 0.7403 \\
\hline 0.4137 & 0.7280 & 0.7286 & 0.7286 & 0.7273 \\
\hline 0.4373 & 0.7065 & 0.708 & 0.7081 & 0.7066 \\
\hline 0.4590 & 0.6755 & 0.6761 & 0.6762 & 0.6746 \\
\hline 0.4784 & 0.6320 & 0.6313 & 0.6314 & 0.6298 \\
\hline 0.4960 & 0.5730 & 0.5723 & 0.5721 & 0.5709 \\
\hline 0.5119 & 0.4990 & 0.4998 & 0.4993 & 0.4987 \\
\hline 0.5265 & 0.4130 & 0.4137 & 0.4130 & 0.4130 \\
\hline 0.5398 & 0.3165 & 0.3176 & 0.3167 & 0.3173 \\
\hline 0.5521 & 0.2120 & 0.2123 & 0.2115 & 0.2125 \\
\hline 0.5633 & 0.1035 & 0.1025 & 0.1020 & 0.1030 \\
\hline 0.5736 & -0.010 & -0.0084 & -0.0082 & -0.0076 \\
\hline 0.5833 & -0.123 & -0.1250 & -0.1239 & -0.1242 \\
\hline 0.5900 & -0.210 & -0.2079 & -0.2058 & -0.2073 \\
\hline
\end{tabular}

TABLE V: COMPARISON OF OPTIMAL PARAMETERS OBTAINED USING THE THREE WOAS

\begin{tabular}{llll}
\hline \hline Parameter & Original WOA & $\begin{array}{l}\text { Tournament } \\
\text { WOA }\end{array}$ & $\begin{array}{l}\text { Rank-based } \\
\text { WOA }\end{array}$ \\
\hline$R_{s e r}(\Omega)$ & 0.0364 & 0.0369 & 0.0357 \\
$R_{s h}(\Omega)$ & 78.6195 & 94.7249 & 72.6882 \\
$I_{p h}(\mathrm{~A})$ & 0.7604 & 0.7589 & 0.7597 \\
$I_{s d l}(\mu \mathrm{A})$ & 0.2940 & 0.1870 & 0.3635 \\
$I_{s d 2}(\mu \mathrm{A})$ & 0.4944 & 0.1455 & 0.0455 \\
$n_{1}$ & 1.4762 & 1.4682 & 1.5100 \\
$n_{2}$ & 1.9984 & 1.5129 & 1.4834 \\
$R M S E$ & $1.23846 \mathrm{e}-003$ & $1.43755 \mathrm{e}-003$ & $\mathbf{1 . 1 8 1 7 3 e - 0 0 3}$ \\
\hline \hline
\end{tabular}

\section{Temperature Effect Analysis of Solar Cells}

From (4), since the net current $\left(\mathrm{I}_{\mathrm{t}}\right)$ is strongly related to the cell temperature $(\mathrm{T})$ with nonlinear relationship, the effect of cell temperature on the I-V and Power-V characteristic curves was further analyzed in this work. The cell temperatures were set to be $25^{\circ} \mathrm{C}, 33^{\circ} \mathrm{C}, 50^{\circ} \mathrm{C}$, and $75^{\circ} \mathrm{C}$. The algorithm used for this study was the proposed rank-based WOA. Fig. 7 shows that the net current in the I-V curve increases significantly when the cell temperature increases. Also Fig. 8 shows that the power-voltage curve increases significantly when the cell 
temperature increases.

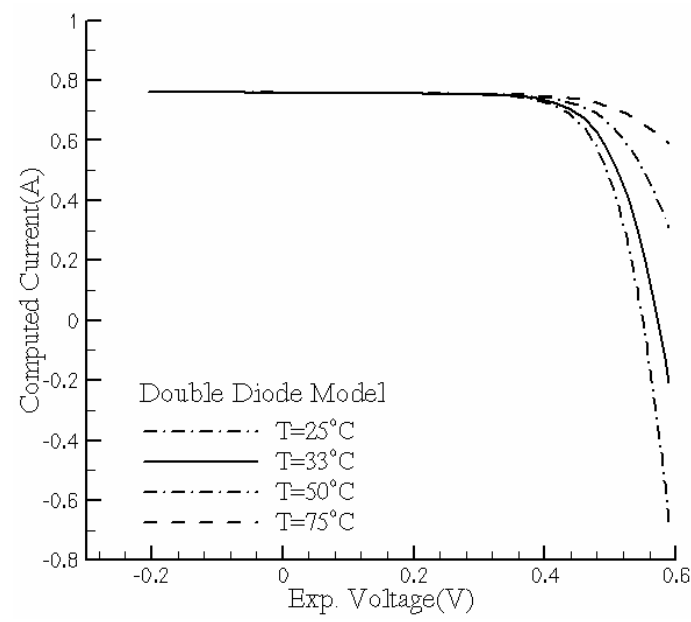

Fig. 7. Effect of cell temperature on I-V curve.

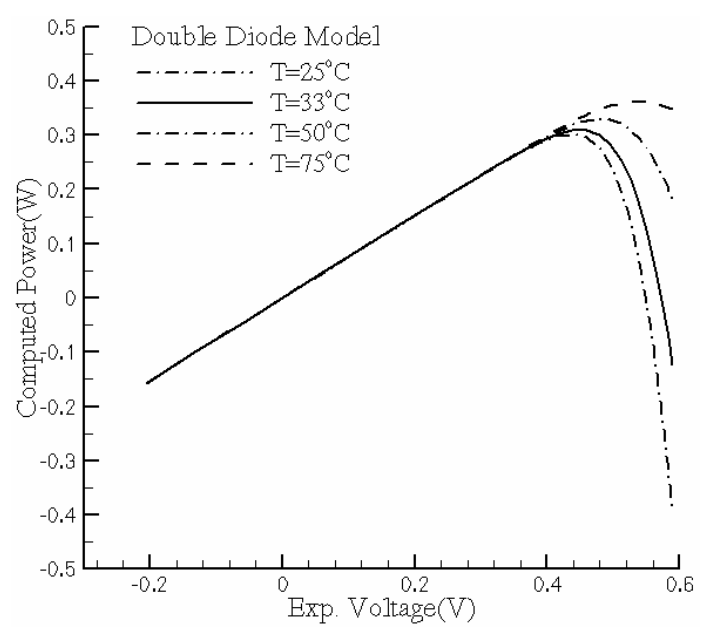

Fig. 8. Effect of cell temperature on Power-V curve.

\section{CONCLUSION}

In this paper, an improved whale swarm algorithm named rank-based WOA was proposed. The nonlinear rank-based selection pressure model was applied to select the agent at the stage of searching for prey in the proposed WOA, rather than random selection approach in the original WOA. The search efficiency and the accuracy of optimal solution using the proposed rank-based WOA were improved through the evaluations of six benchmark functions in the medium- and large-scale sizes of space. Furthermore, the original, tournament selection based, and rank-based WOAs were applied to the parameter estimation of the solar cells. Double diode model was used for simulating the electronic circuits of solar cells. The results showed that the net currents computed by the three WOAs were quite close to the experimental data. The rank-based WOA was superior to the original and tournament WOAs in terms of convergence rate and solution accuracy. Further analysis of the cell temperature effect showed that when the operating temperature of the solar cell increases, the efficiency of the I-V and Power-V characteristic curves of the solar cell will be improved.

\section{REFERENCES}

[1] Energy Trend. [Online]. Available: https://technews.tw/2018/03/19/solar-energy- device-capacity-2017/

[2] A. Alireza and R. Alireza, "Parameter identification for solar cell models uesing harmony search-based algorithms," Solar Energy, vol. 86, issue 11, pp. 3241-3249. Nov. 2012.

[3] T. Easwarakhanthan, J. Bottin, I. Bouhouch, and C. Boutrit, "Nonlinear minimization algorithm for determining the solar cell parameters with microcomputers," Solar Energy, vol. 4, issue 1, pp. 1-12. Jan. 1986.

[4] A. Ortiz-Conde, F. J. Garcia Sanchez, and J. Muci, "New method to extract the model parameters of solar cells from the explicit analytic solutions of their illuminated I-V characteristics," Solar Energy Materials and Solar Cells, vol. 90, issue 3, pp. 352-361, Oct. 2006.

[5] D. Chan, J. R. Phillips, and J. Phang, "A comparative study of extraction methods for solar cell model parameters," Solid-State Electron, vol. 29, issue 3, pp. 329-337, 1986.

[6] J. A. Jervase, H. Bourdoucen, and A. Al-Lawati, "Solar cell parameter extraction using genetic algorithms," Measurement Science and Technology, vol. 12, issue 11, pp. 1922-1925, Oct. 2001.

[7] H. Wei, J. Cong, X. Lingyun, and S. Deyun, "Extracting solar cell model parameters based on chaos particle swarm algorithm," in Proc. International Conference on Electric Information and Control Engineering, Wuhan, China, April 15-17, 2011.

[8] D. Oliva, E. Cuevas, and G. Pajares, "Parameter identification of solar cells using artificial bee colony optimization," Energy, vol. 72, pp. 93-102, Aug. 2014.

[9] D. Oliva, M. Abd El Aziz, and A. E. Hassanien, "Parameter estimation of photovoltaic cells using an improved chaotic whale optimization algorithm," Applied Energy, vol. 200, pp. 141-154, Aug. 2017.

[10] S. Mirjalili and A. Lewis, "The whale optimization algorithm," Advances in Engineering Software, vol. 95, pp. 51-676, May 2016.

[11] W. Shockley, "The theory of p-n junctions in semiconductors and p-n junction transistors," The Bell System Technical Journal, vol. 28, issue 3, 435-489, Jul. 1949.

[12] M. M. Mafarja and S. Mirjalili, "Hybrid whale optimization algorithm with simulated annealing for feature selection," Neurocomputing, vol. 28, no. 3, pp. 1-11, Oct. 2017.

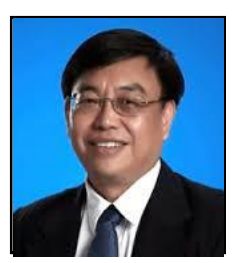

Jeng-Shyang Pan received the Ph.D. degree in electrical engineering from the University of Edinburgh, U.K. in 1996. Currently, he is a professor in Harbin Institute of Technology Shenzhen and Fujian University of Technology. Dr. Pan is also the dean of College of Information Science and Engineering, Fujian University of Technology. His current research interests include soft computing, information security, big data mining and signal processing.

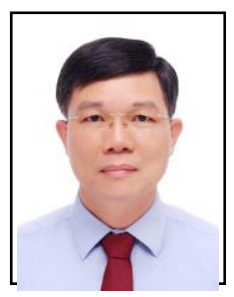

Jenn-Long Liu received his $\mathrm{PhD}$ degree in aeronautics and astronautics from National Cheng Kung University, Taiwan in 1991. He is a professor at the Department of Information Management of I-Shou University, Kaohsiung, Taiwan. He also had been a visiting scholar at University of California, Riverside (UCR) and Illinois State University (ISU) in 2010 and 2014, respectively. His current research interests include artificial intelligence, evolutionary computation, data mining, and big data analysis. Dr. Liu is a life member of the Taiwanese Association for Artificial Intelligence (TAAI).

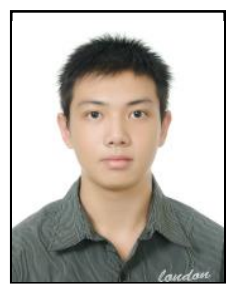

En-Jui Liu received his MS degree in power energering from National Central University, Taiwan in 2015. Currently, he is a doctoral student at the Department of Power Mechanical Engineering, National Tsing Hua University, Taiwan. His current research interests include swarm-based metaheuristic optimization algorithms, fuel cell, energy system, greenergy engineering. 